\title{
DIE GRÜNDUNGSURKUNDE DES HOSPITALS NOTRE-DAME-DES-FONTENILLES IN TONNERRE
}

\begin{abstract}
Die Urkunde, mit der Papst Bonifaz VIII. im Jahre 1297 die Stiftung des Hospitals Notre-Dame-des-Fontenilles in Tonnerre bestätigte, fällt dem Betrachter zunächst einmal wegen ihren Maßen auf. Sie gehört zweifellos zu den größten und längsten Papsturkunden, die je geschrieben wurden. Der Historiker schätzt natürlich vor allem ihre präzisen und detaillierten Angaben über das Aussehen, die Struktur und die Funktion der neuen Anstalt. Da dieses außerordentliche Dokument nur in einem mangelhaften Druck von 1630 vorliegt, wird es an dieser Stelle neu ediert.
\end{abstract}

\section{Edition}

Orvieto, 1297 September 5 Bonifaz VIII. bestätigt auf Bitten Margaretas, Königin von Sizilien und Gräfin von Tonnerre, des Meisters und der Brüder des Hospitals von Tonnerre die am 9. April 1293 erfolgte Stiftung des Hospitals und läßt die Stiftungsurkunde inserieren.

A Tonnerre, Archives de l'Hôpital Notre-Dame des Fontenilles, $H$ supplément 2840/ A I (Original mit Bleisiegel an Seidelbündel); der Edition liegt der Mikrofilm der Archives départementales in Auxerre zugrunde; $\boldsymbol{B}$ Città del Vaticano, Archivio Segreto Vaticano, Reg. Vat. 48 fol. $310 \mathrm{v}-314 \mathrm{r} \mathrm{Nr}$. CCCCI.

Druck: David Andry, Chartres (!) et titres anciens des habitans de Tonnerre, Auxerre 1630. S. $105-122($ nach $A)$.

Regest: August Potthast, Regesta pontificum Romanorum, 2 Bde., Berlin 1875, hier Bd. $2 \mathrm{Nr}$. 24569 mit dem falschen Incipit Cum a nobis; Les registres de Boniface VIII (I294-1303). Recueil des bulles de ce pape publiées ou analysées d'après les manuscrits originaux des Archives du Vatican par Georges Digard et al., 4 Bde., Paris 1884-1939, hier Bd. I Sp. 813f. Nr. 2118.

Bonifacius episcopus servus servorum Dei ${ }^{a}$. Dilectis filiis .. magistro et fratribus hospitalis sancte Marie de castro Tornodori ad Romanam ecclesiam nullo medio pertinentis Lingonensis diocesis salutem et apostolicam benedic-

\footnotetext{
${ }^{a}$ Bonifatius - Dei fehlt B
} 
tionem ${ }^{\mathrm{a}}$. Hiis, que ${ }^{\mathrm{b}}$ a Christi fidelibus pro divini amore nominis misericorditer peraguntur, libenter adicimus apostolici muniminis firmitatem, ut intemerata consistant, que nostro fuerint presidio communita. Sane petitio vestra nobis exhibita continebat, quod carissima in Christo filia nostra Margareta regina Sicilie illustris comitissa Tornodori relicta clare memorie C. ${ }^{1}$ Sicilie regis vidua provide sua novissima memorans et attendens, quod bona sua non poterat melius propriis utilitatibus applicare quam si de ipsis aliqua ${ }^{\mathrm{c}}$ in honorem bonorum omnium largitoris expenderet, de cuius ${ }^{d}$ recepit $^{\mathrm{e}}$ habundanti ${ }^{\mathrm{f}}$ gratia universa, hospitale vestrum sancte Marie de castro Tornodori ad opus infirmorum et pauperum necnon capellam seu oratorium et altaria, ut ibidem iugiter divina officia celebrentur, de bonis propriis construens sive construi faciens quasdam domos, terras, possessiones, redditus, iura, iurisdictiones et nonnulla alia bona ad ipsam spectantia hospitali predicto in perpetuum pia et provida deliberatione contulit et donavit vobis et eidem hospitali quasdam libertates et immunitates nichilominus concedendo ac etiam quasdam ordinationes faciendo in dicto hospitali perpetuis temporibus observandas, prout in patentibus litteris inde confectis predicte regine sigillo munitis plenius continetur. Nos itaque predicte regine ac vestris supplicationibus inclinati, collationes, donationes, concessiones ${ }^{\mathfrak{g}}$, ordinationes huiusmodi et quecumque super predictis a prefata regina facta ${ }^{\mathrm{h}}$ sunt in eisdem contenta ${ }^{\mathrm{i}}$ litteris rata et grata habentes ac defectum, si quis ex eo fuerit in premissis, quod sine assensu venerabilis fratris nostri .. episcopi Lingonensis, a quo eadem regina comitatum Tornodori in feudum tenere dicitur, prefatum hospitale vestrum pro maiori parte de bonis dicti comitatus fundatum esse noscitur ${ }^{k}$ et dotatum, supplentes de apostolice plenitudine potestatis ea auctoritate apostolica ex certa scientia confirmamus et presentis scripti patrocinio communimus, tenorem predictarum litterarum de verbo ad verbum presentibus inseri facientes, qui talis est:

In nomine patris et filii et spiritus sancti amen. Nos Margareta Dei gratia lerusalemitana ${ }^{\mathrm{l}}$ et Sicilie regina Tornodori comitissa notum facimus universis presentes litteras inspecturis, quod nos attendentes verba evangelii, quo legitur „Estote misericordes sicut et pater vester misericors est“, advertentes etiam

${ }^{a}$ Dilectis - diocesis Rubrik B, salutem - benedictionem fehlt B

${ }^{b}$ fehlt B

${ }^{c} \mathrm{~B}$ aliquod

cuius fehlt B

${ }^{e} \mathrm{~B}$ receptis

$f_{\text {sic }}$

${ }^{8} \mathrm{~B}$ folgt $e t$

' facta fehlt B

${ }^{i} \mathrm{~B}$ contempta

${ }^{j}$ A fuit

${ }^{k} \mathrm{~B}$ dicitur

'A Ierlm, B Iersitan 
misericordiam, quam pater noster creator omnium fecit in nos in creatione, redemptione et bonorum temporalium largitione, ne valeamus ingrate iudicari, quod absit, compatientes Christi pauperibus volentesque sancto Dei evangelio non quantum debemus, sed quantum possumus obedire, desiderantes in eisdem misericordiam extendere temporalem ${ }^{\mathrm{a}}$, cupientes remunerationem acquirere $^{b}$, que in evangelio promittitur facientibus misericordiam scilicet gloriam sempiternam, et evitare penam, que infligitur ${ }^{c}$ misericordiam non agentibus ignem videlicet sempiternum, hospitale seu domum Dei fundamus et constituimus apud Tornodorum in vico seu loco dicto Fontenilles. Et ad ipsum hospitale seu domum Dei fundandum et edificandum loca damus et concedimus infrascripta videlicet vicum de Tornodoro dictum de Tonneleria a porta vadi existentis ante domum, que fuit Lamberti dicti Trinchet, usque ad domum monialium de ${ }^{\mathrm{d}}$ Iuilliaco sitam in fine vici dicti Fontenilles, prout mete important, a ripparia ${ }^{e}$ Tornodori usque ad ipsam domum et a dicto fine vici de Fontenilles, prout mete important, usque ad portam, que dicitur porta de Fontenilles, et ab ipsa porta de Fontenilles, prout mete important, usque ultra portam dictam de Rubeo Monte et de ultra portam ipsam, prout mete important, usque ad filum ripparie ${ }^{f}$. Quas omnes metas in dictis locis poni fecimus et infigi. In quibus locis construi volumus et edificari capellam et oratorium cum quatuor altaribus in eisdem, maius videlicet altare in honore beate Marie virginis, secundum in honore beati Iohannis baptiste, tertium in honore beate Marie Magdalene $^{\mathfrak{g}}$ et quartum in honore beate Elisabeth de Duringo. Volumus etiam cimiterium fieri pertinens ad hospitale predictum et domos edificari, in quibus magister, fratres et sorores eiusdem domus maneant, pauperes hospitentur et bona dicti hospitalis propria conserventur. Que loca omnia cum proprisiis ${ }^{\text {h }}$ omnibus eorundem, prout de metis ad metas in circuitu protenduntur, et infra metas et sine metis longe lateque integraliter se comportant, volumus ad dictum hospitale seu domum Dei ex nunc in perpetuum iure hereditario pertinere una cum dominio et omnimoda iustitia dictorum locorum tali tamen conditione adhibita pro nobis et successoribus nostris comitibus Tornodori, quod, si per gentem sive servientem dicti hospitalis aliquis vel aliqui malefactores deprehensi fuerint infra metas proprisie ${ }^{i}$ dicti hospitalis, qui propter sui maleficium seu maleficia infra ipsas metas vel alibi perpetrata morte cruciari, membro mutilari vel

\footnotetext{
${ }^{a} \mathrm{~B}$ corporalem

${ }^{b} \mathrm{~B}$ accipere

${ }^{c} \mathrm{~B}$ infligit

d de fehlt $\mathrm{B}$

${ }^{e} \mathrm{~B}$ riparia

${ }^{5} \mathrm{~B}$ riparie

${ }^{g}$ A und B Magdalenes

${ }^{h} \mathrm{~A}$ und $\mathrm{B}$ porprisiis

'A und B porprisie
} 
exulari seu banniri meruerint, prepositus noster de Tornodoro, qui pro tempore fuerit, vel eius locum tenens, postquam ex parte dicti servientis super hoc fuerit requisitus, statim ad unam de metis dicti hospitalis festinanter accedens a manibus dicti servientis ipsum vel ipsos malefactores in tunica vel in camisia tradentes et liberantes recipiat ${ }^{a}$ extra terram et metas ipsius hospitalis. Si vero malefactor vel malefactores predicti, antequam tradantur dicto preposito aliquo casu manus evaserint vel effugerint gentis vel servientis hospitalis predicti, nos super hoc heredes vel successores nostri vel gens nostra magistrum, fratres, gentem et servientem hospitalis predicti molestare vel occasionare non poterimus nec aliquid propter hoc ab eisdem petere vel exigere quoquomodo, nisi tantummodo iuramentum a dicto serviente prestandum super eo, quod prece vel pretio seu modo quolibet alio evasioni vel fuge malefactoris seu malefactorum huiusmodi dolum non adhibuerit neque fraudem, quod etiam iuramentum ad requisitionem dicti prepositi fieri volumus et prestari; quod, si dictus serviens recusans iuramentum non fecerit, si frater existat, magister eum carceri mancipatum puniat per duos menses cum pane et aqua sine spe eiusdem officii infra biennium resumendi; si vero sit extraneus, ab officio et hospitali propter hoc expellatur et extra terram et metas ipsius hospitalis ducatur, ut ibidem prepositus Tornodori eum recipiat, si sua crediderit interesse pro ${ }^{b}$ faciendo de ipso, prout viderit faciendum. Capellam autem, oratorium et domos, in quibus magister, fratres, sorores, et pauperes iacebunt, eximimus et liberos ac immunes esse volumus ab omni potestate, iurisdictione et iustitia temporali nostra et heredum ac successorum nostrorum, volentes ipsa loca omnia eorumque iustitiam ipsam hospitale, magistrum, fratres et sorores eiusdem cum habitatoribus et omnibus bonis suis in comitatu Tornodori constitutis esse perpetuo de garda et speciali custodia nostra et comitis Tornodori pro tempore affuturi $^{\mathrm{c}}$. Qui comes non possit emptione, venditione, partagio, escambio seu permutatione, donatione aut alia quacumque de causa ponere extra manum suam dictam gardam et custodiam dicti hospitalis bonorum et pertinentiarum eiusdem, et si contingeret, quod aliquis causa pietatis et devotionis conferret se et sua Deo et hospitali predicto et de licentia magistri extra hospitale predictum in comitatu Tornodori et aliis terris quibusque alicubi moraretur, volumus, precipimus et ordinamus, quod persona ipsius et bona eadem immunitate gaudeant sicut unus de fratribus hospitalis predicti. In quo hospitali seu domo Dei fieri volumus septem opera misericordie scilicet famelicos cibari, sitientes potari, peregrinos et hospites recipi et hospitari, nudos vestiri, infirmos visitari, incarceratos confortari et mortuos sepeliri. Et quia voluntas nostra parum aut

\footnotetext{
a A tradentis et liberantis recipiet

${ }^{b}$ pro fehit B

${ }^{c} \mathbf{B}$ afuturi
} 
nichil prodesset, nisi esset, qui et de quo eam effectui manciparet ${ }^{2}$ et predicta misericordie opera adimpleret ${ }^{b}$, nos cultum cupientes ampliare divinum volumus, ibi magistrum institui personam litteratam cum quatuor capellanis et quatuor pueris clericis, qui caste et continenter ibi vivant et celebrent ad minus singulis diebus ibidem duas missas, unam videlicet de sancto spiritu, quamdiu vixerimus, et post mortem nostram pro defunctis, nisi festum fuerit annale, et aliam de virgine Maria vel de die seu de festo, si, quod evenerit, ipsa die vel de quocumque maluerint, secundum quod viderint expedire; volentes, quod magister, fratres et sorores ipsius hospitalis anniversarium felicis recordationis domini K. quondam Ierosolimitani ${ }^{c}$ et Sicilie regis consortis nostri carissimi singulis annis die obitus sui sollempniter celebrent et post decessum nostrum anniversarium nostrum una cum suo ipsa die obitus sui et iterum suum una cum nostro die obitus nostri in eodem hospitali singulis annis celebrare sollempniter tenebuntur. Magister cum quatuor capellanis ${ }^{d}$ et quatuor pueris surgent singulis diebus ad matutinas, si comode possint, et dicent missam et horas canonicas alta voce cantando ita, quod possint ab infirmis audiri. Qui magister et capellani in domo ipsa et capella habebunt semper et deferent ${ }^{c}$ superpellicium vel rochetum, nisi forte ipsis in domo seu proprisia ${ }^{f}$ pro temporali comodo dicte domus manualiter negotiari contingat. Et, cum domum exierint, superpellicia vel capas ${ }^{g}$ clausas sive houcias ${ }^{\text {h }}$ vel supertunicalia ferent de camellino vel panno nigro, exeuntes autem villam capas ${ }^{i}$ vel houcias deferent supradictas. Dicti autem pueri instruentur ibidem in gramatica et musica horis canonicis insistentes. Magister vel aliquis capellanus vice eius, cum infirmi ad domum venerint, visitabit eosdem inducendo, quod confiteantur peccata, et cogitent, qualiter vixerint ${ }^{k}$ in seculo, dicens et instruens, quod, qui in peccato mortali decedit, in igne erit perpetuo, qui vero sine peccato erit cum angelis in gloria sempiterna; quibus infirmis sacramenta ecclesiastica, cum opus fuerit, ministrabit et omnibus de ipso hospitali penitentiam, eucharistiam et extremam unctionem. In quo hospitali mulieres parturientes custodiantur et releventur, quibus et aliis infirmis ibidem affluentibus in pastu et potu necessaria ministrentur, prout videbitur expedire. Et decedentes in domo ipsa magister sive

\footnotetext{
${ }^{a} \mathrm{~B}$ efectui maciparet

${ }^{b} \mathrm{~B}$ adimplere

'A Ierlm

${ }^{d} \mathrm{~B}$ cappellanis

${ }^{e} \mathrm{~B}$ defferent

${ }^{f} \mathrm{~A}$ und $\mathrm{B}$ porprisia

${ }^{8} \mathrm{~B}$ cappas

${ }^{\text {}} \mathrm{B}$ houcia

${ }^{i} \mathrm{~B}$ cappas

${ }^{j} \mathrm{~B}$ houncias

${ }^{k} \mathrm{~B}$ viserint
} 
aliquis de capellanis predictis in dicto cimiterio faciet honorifice sepeliri. Iterum in dicto hospitali instituetur quedam matrona magistra cum duodecim mulieribus, que custodient et relevabunt infirmos et custodient et servabunt linteamina, cultras, plumaria et coopertoria ${ }^{a}$ munda ad opus pauperum et infirmorum. Et erunt dicte mulieres in domo indute de camellino vel aliquo panno nigro velum habentes et desuper aliquo rocheto albo, quamdiu suum officium exercebunt. Magister et magistra predicti prestabunt in suis institutionibus iuramenta, quod negotia et administrationem bonorum dicti hospitalis, prout intererit, utriusque ipsorum gerent utilius quam poterunt, exercebunt fideliter et curabunt. Et, quod erga pauperes dicti hospitalis se gerent misericorditer et habebunt, magister ministrabit eidem magistre singulis ebdomadis videlicet de pane et denariis qualibet ${ }^{b}$ die sabati mane pro dimidia ebdomada panem unius sextarii frumenti et triginta solidos monete currentis pro tempore apud Tornodorum et pro residuo ebdomade totidem panis, frumenti et denariorum qualibet die martis ${ }^{\mathrm{c}}$ mane et de vino singulis diebus sex sextarios ${ }^{\mathrm{d}}$. Et hec omnia pro ministrando pauperibus et hospitibus hospitalis predicti, prout expedit et decebit. Item magister eidem magistre carnes salsatas pro omnibus necessariis pro pauperibus dicti hospitalis ministrabit sufficienter, secundum quod viderit expedire. Et, si omnibus hiis rite consumptis aliquid pro necessariis ebdomade adimplendis defuerit, magister illud ad petitionem magistre supplebit. Si vero aliquid omnibus hiis rite peractis ${ }^{\mathrm{e}}$ residui fuerit, illud residuum magistra distribuet pauperibus incarceratis et aliis in villa Tornodori, secundum quod viderit expedire. Item magister solutis omnibus expensis et missionibus dicti hospitalis et pertinentiarum eiusdem et munitionibus et omnibus ${ }^{f}$ necessariis pro dicto hospitali et ${ }^{\mathrm{g}}$ eiusdem pertinentiis factis et retentis pro uno anno tunc futuro, si quid de redditibus et exitibus residuum fuerit in dicto hospitali cum pertinentiis ${ }^{\mathrm{h}}$, secundum providentiam et industriam dicti magistri de consilio aliquorum bonorum pauperibus erogetur. Pauperes autem, qui in dicto hospitali de lecto surgentes egritudinis convaluerint, ne aliqui sanitati restituti ${ }^{i}$ prenimis festina recessione recidivum patiantur, septem diebus sani in domo sustententur et nudi non exeant, sed magister eidem magistre petenti ministret, unde ipsa camisiam, tunicam et sotulares ${ }^{j}$, prout expedit, convalescenti ministret.

\footnotetext{
${ }^{a}$ B coopatoria

${ }^{b} \mathrm{~B}$ quamlibet

${ }^{c} \mathrm{~B}$ folgt de

${ }^{d} \mathrm{~B}$ sestarios

${ }^{e} \mathrm{~B}$ pactis

${ }^{6} \mathrm{~B}$ folgt aliis

$\boldsymbol{g}_{\text {et fehlt B }}$

${ }^{h}$ B folgt suis

${ }^{i} \mathrm{~B}$ restuti

${ }^{j}$ B sotelares
} 
Volumus, quod numerus capellanorum ${ }^{\mathrm{a}}$ et puerorum cum magistro existentium et mulierum cum magistra manentium viginti non excedat scilicet capellanorum quatuor, puerorum quatuor et duodecim mulierum, nisi forsitan necessitate urgente et pietate seu misericordia suadente, ita tamen, quod dicta portio eidem magistre pro pauperibus facienda nullatenus propter hoc diminuatur. Qui capellani ${ }^{\mathrm{b}}$ et pueri, fratres, magistra et sorores mandatum dicti magistri et eius vices gerentis ipso magistro absente tenebuntur observare, ac etiam mulieres in suis officiis exercendis dicte magistre mandatum observabunt ${ }^{c}$, quorum quarumque omnium correctio et omnimoda iurisdictio ad magistrum pertinebit. Magister familiam habeat, fratres et alios, secundum quod pro domibus et grangiis et aliis hospitalis predicti ${ }^{d}$ rebus et negotiis viderit expedire, et magistra duas ancillas vel plus, si necesse fuerit et videbitur expedire. Magister vero recipiet capellanos, pueros, fratres et sorores ydoneos moribus et etate, secundum quod viderit expedire hospitali predicto, nostra auctoritate et consensu intervenientibus. Post decessum autem nostrum premissa faciet de consilio comitis et successorum nostrorum Tornodori, nec potuerunt dictus comes et successores nostri factum receptionis dicti magistri aliquatenus impedire, nisi manifestissima et apparenti iusta causa. Qui capellani et fratres, magistra et sorores professionem facient dicto magistro sollempniter et in professionibus suis magistro promittent obedientiam, continentiam et proprii carentiam cum stabilitate propositi in eodem loco perpetuo permanendi ${ }^{\mathrm{e}}$. Et quod vivere teneantur secundum statuta et ordinationes per nos editas et factas ac eas inviolabiliter observare, prout tam in presentibus quam in aliis nostris litteris conficiendis super hoc et sigillo nostro sigillandis videbitur plenius contineri. Magister expensas faciet in victualibus et omnibus necessariis sufficientes pro se, capellanis, pueris, fratribus et tota familia sua, prout viderit expedire, et dicte magistre pro se et mulieribus suis et etiam pedissecis ${ }^{f}$ sufficienter tales et consimiles ministrabit expensas. Item magister faciat luminaria in capella seu oratorio videlicet qualibet die, dum hore cantabuntur ${ }^{g}$ et misse celebrabuntur, ad maius altare duo cerei erunt ardentes et ad quodlibet aliorum altarium unus, dum missa celebrabitur in eodem, et in festis duplicibus ${ }^{\mathrm{h}}$ et annalibus ad omnia altaria duplicentur'. Magister et magistra vestes novas bis habebunt in anno

\footnotetext{
${ }^{a} \mathrm{~B}$ cappellanorum

${ }^{b} \mathrm{~B}$ cappellani

'A servabunt

${ }^{d} \mathrm{~B}$ hospitalitatis predictis

${ }^{e} \mathrm{~B}$ permanendum

${ }^{\prime}$ sic

${ }^{8} \mathrm{~B}$ cantanbuntur

${ }^{\text {h A dupplicibus }}$

${ }^{i}$ A dupplicentur
} 
de camellino ${ }^{a}$ vel panno nigro et pennas de cuniculis vel agniculis videlicet magister singulis annis ad festum Omnium sanctorum tunicam, supertunicale et corsetum ${ }^{b}$, item ad Pascha ${ }^{c}$ totidem. Item cappam ${ }^{d}$ unam et houciam unam per annum. Magistra vero singulis annis ad eadem festa tunicam et supertunicale nova et pellicium unum de cuniculis per annum. Capellani vero, pueri et fratres et sorores vestes habebunt novas semel in anno videlicet quilibet ipsorum tunicam et supertunicale fourratum ad festum Omnium sanctorum et ad Pascha quilibet unum supertunicale fourratum et quilibet fratrum singulis annis unam houciam. Item magister alii familie vestes, calciamenta et alia, prout expedire viderit, ministrabit. Quando autem magistrum et magistram mori contingerit $^{\mathrm{e}}$ vel alias deficere, substitueret ${ }^{f}$ loco dicti magistri aliquis de dictis capellanis et loco magistre aliqua de dictis mulieribus, si ydonei fuerint, alioquin alie persone sufficientes et honeste, secundum quod dictus comes bona conscientia expedire videbit hospitali predicto, cuius conscientiam super hiis penitus oneramus ${ }^{\mathrm{g}}$. Quamdiu ${ }^{\mathrm{h}}$ vero vixerimus, instituemus magistrum et magistram in hospitali predicto et eos destituemus et amovebimus' cum iusta ${ }^{j}$ causa de adminstrationibus suis, si deceat et viderimus expedire, et eos etiam in hiis, que ad administrationem bonorum dicti hospitalis pertinent, corrigemus. Et post decessum nostrum ad dictum comitem Tornodori et successores eius dominos Tornodori dictorum magistri et magistre institutio et destitutio vel amotio $^{k}$ a suis administrationibus et correctio pertinebit. Dictos tamen magistrum et magistram dictus comes et successores sui, ut dictum est, absque iusta, notoria, manifesta et evidenti causa non poterunt destituere nec etiam amovere. Damus autem et concedimus pro hedification $\mathrm{e}^{\mathrm{m}}$ dicti hospitalis seu domus Dei muros nostros ville Tornodori existentes in locis supradictis concessis a nobis in ipsa edificatione et fundatione, ad edificandum ipsum hospitale, prout ipsa loca infra metas et ipse mete circumquaque important, volentes et plenius concedentes ipsa loca omnia et singula, prout in eorundem toto circuitu tam de dictis metis ad metas quam etiam sine metis et usque ultra dictam portam dic-

\footnotetext{
a camelino

${ }^{b} \mathrm{~B}$ corsettum

' B pasca

${ }^{d}$ sic

'A contigerit

${ }^{f}$ B substituetur

${ }^{g}$ B honeramus

${ }^{\mathrm{h}} \mathrm{B}$ quadiu

'A ammovebimus

${ }^{j} \mathrm{~B}$ vre (!)

${ }^{k}$ sic

${ }^{\prime}$ A ammovere

${ }^{m}$ sic
} 
tam de Rubeo ${ }^{a}$ Monte et inde ad rippariam ${ }^{b}$, ut dictum est, integraliter se comportant, claudi posse et debere muris altis, latis et amplis munitis ${ }^{c}$ turribus et crenellis, prout nobis, quoad vixerimus, et post mortem nostram executoribus nostris vel duobus aut uni ipsorum et dicto magistro et ipsis executoribus mortuis vel non presentibus dicto magistro placuerit et videbitur expedire absque ulla contradictione et impedimento vel molestatione eidem hospitali super hiis aliquatenus inferendis a successoribus nostris comitibus seu dominis Tornodori, qui pro tempore erunt, vel eorum gente seu officialibus et aliis quibuscumque. Ut autem predicta misericordie opera in dicto hospitali competenter fieri valeant et compleri in dicti hospitalis seu domus Dei fundatione perpetua damus et perpetuo concedimus eidem una cum supradictis res et bona omnia infrascripta videlicet centum modios vini annui redditus, quos assignamus et affidamus ${ }^{d}$ eidem hospitali super decimam nostram vinorum de Tornodoro; quos centum modios vini percipi volumus et levari per magistrum dicti hospitalis vel eius mandatum singulis annis de grossioribus summis vini boni et bene sufficientis de decima nostra de Tornodoro, antequam cellerarius Tornodo$\mathrm{ri}^{\mathrm{e}}$ vel quicumque $\mathrm{f}^{\mathrm{f}}$ alius levator ${ }^{8}$ decime vinorum eiusdem levet aliquid de eisdem. Quod, si forte dicta vina decime Tornodori aliquo tempore non suffecerint ad perceptionem integram dictorum centum modiorum vini, dictus magister residuum, quod deerit, levabit per se vel per alium in decima vinorum de Espinolio et de locis aliis propinquioribus, ubicumque voluerit et viderit comodius esse sibi. Quod, si in $^{\mathrm{h}}$ aliquo anno tanta sterilitas sive defectio, quod absit, fuerit in dictis vinis, quod tota decima vinorum de Tornodoro, de Espinolio et aliis villis et locis predictis ad solutionem dictorum centum modiorum vini non suffecerint, illud, quod defuerit, cum cautione ydonea et sufficienti expectetur et suppleatur anno immediate sequenti ex vino, si fuerit, alioquin, si non fuerit, in valore seu pretio, quo tunc erit. Volumus etiam et districte precipimus, quod cellerarius Tornodori et quicumque alius, quem institui pro tempore contigerit ad levandum dictam decimam, iuret in institutione sua super sancta Dei evangelia coram domino magistro vel eius mandato, quod quamproximis singulis annis decimam ipsam iactaverit, taxaverit, summaverit vel signaverit statim, antequam levet aliquid de eisdem summis vinorum dicte decime, et nomina debitorum eiusdem dicto magistro vel eius mandato tradet et

\footnotetext{
${ }^{a} \mathrm{~B}$ folgt de

${ }^{b} \mathrm{~B}$ ripariam

${ }^{c} \mathrm{~B}$ munitibus

${ }^{d} \mathrm{~B}$ affidemus

- Tomodori fehlt B

${ }^{\prime} \mathrm{B}$ quacumque

${ }^{8} \mathrm{~B}$ folgt $e t$

${ }^{h}$ in fehlt B

${ }^{i} \mathrm{~B}$ vacaverit
} 
liberabit in scriptis, ut idem magister levet vel levari faciat dictos centum vini modios annis singulis modo et forma supradictis sine contradictione vel molestatione aliqua vel impedimento dicto magistro vel eius mandato super hoc inferendis. Damus etiam eidem hospitali, quicquid habemus in minagio et rotagio Tornodori scilicet ${ }^{\mathrm{a}}$ in demanio, et triginta libras annui ${ }^{\mathrm{b}}$ redditus super festagium dicte ville Tornodori dicto magistro vel eius mandato annuatim solvendas a collectore dicti festagii de denariis eiusdem festagii ${ }^{c}$ primo levatis singulis annis per iuramentum collectoris ipsius festagii, quod super hoc ab eodem collectore in institutione sua dicto magistro vel eius mandato ad requisitionem ipsius fieri volumus et prestari. Damus etiam eidem hospitali gardas seu custodias vinearum Tornodori et pertinentiarum cum emolumentis salvis emendis prisiarum, que erunt domino Tornodori et servienti earumdem vinearum, sicut consuetum est. Damus ${ }^{d}$ iterum eidem hospitali de ${ }^{e}$ ripparia nostra de Tornodoro illud totum, quod est et protenditur ascendendo a pontibus lapideis sitis uno videlicet ante domum religiosorum ${ }^{f}$ de Fonteneto et alio ante domum, que fuisse dicitur Lamberti dicti Trinchet, usque ad rippariam ${ }^{g}$ religiosorum beati Petri Melugdensis. Item redditum illum, in quo piscatores ad tribulam et ad iunchias ${ }^{\mathrm{h}}$ nobis $\mathrm{s}^{\mathrm{i}}$ annis singulis tenentur pro piscando in ripparia Tornodori, cum omni redditus emolumento predicti. Item prata, que acquisivimus de novo in finagio Tornodori contigua ripparie supradicte ex parte una et rebus infrascriptis ex altera videlicet orto religiosorum sancti Michaelis, clausello Galteri ${ }^{j}$ de Meso predicti, qui clausellus fuisse dicitur defuncti Regnaudi de Moris, prato Iohannis dicti Aiget et Henrieti ${ }^{\mathrm{k}}$ carnificis, prato curati ecclesie beate Marie Tornodori, prato Hugueneti de Melisiaco ${ }^{1}$ clerici et prato eiusdem Galteri de Meso. Damus iterum eidem hospitali boscos et nemora nostra de Evigiaco et de Monte Cesino. Item vineas nostras de Tornodoro videlicet clausum dictum de Denemerre circumquaque contiguum rebus infrascriptis videlicet vinee Petri dicti de Sancto Antonio, vinee capituli sancti Petri, quam tenere dicitur Bartholomeus thesaurarius, vinee ${ }^{\mathrm{m}}$ Iohannis dicti Tiressin ${ }^{\mathrm{n}}$, vinee dicti

\footnotetext{
${ }^{a} \mathrm{~B}$ silicet

${ }^{b} \mathrm{~B}$ anui

c solvendas - festagii auf Rasur A

${ }^{d} \mathrm{~B}$ folgt etiam

ede fehlt A

${ }^{f} \mathrm{~B}$ so von religiosorum interlinear

${ }^{g}$ B ripariam

${ }^{h} \mathrm{~B}$ iuncias

${ }^{i}$ nobis fehlt B

${ }^{j} \mathrm{~B}$ Galtero

${ }^{k}$ B Herrici

'B Mesiliaco

${ }^{m} \mathrm{~B}$ vine

${ }^{n}$ B Treesin
} 
Taperiau ${ }^{a}$ de claustro, vinee liberorum Henrieti ${ }^{b}$ dicti Nico, vinee Garneri ${ }^{c}$ clerici, vinee clausarii Ade Espungoti ${ }^{\mathrm{d}}$, vinee Agneti de Vivariis ${ }^{\mathrm{e}}$, vinee Henrieti dicti Leconversat et vinee beati Antonii. Et clausum nostrum dictum de Mortier circumquaque contiguum vineis et rebus infrascriptis, scilicet ${ }^{f}$ vinee sancti Aniani, vinee Garneri dicti Carpentarii, vinee Guidonis generi ${ }^{\mathrm{g}}$ dicti Challe, vinee $^{\text {h }}$ fratrum militie templi de Marchessoi et vinee ${ }^{\mathrm{i}}$ communi de subtus. Item damus eidem hospitali aboonamenta ${ }^{j}$ de Sanctis Virtutibus et de Merroliis. Item homines et terram, quos acquisivimus ab heredibus defuncti Gaufridi de Sommexo quondam ${ }^{k}$ militis, apud Ruffeyum et alibi. Item salicetum situm in villa Tornodori, quod acquisivimus a relicta defuncti ${ }^{1}$ Godiotis. Item domum fortem et quicquid acquisivimus ab heredibus defuncti Robini de Argentolio apud Argentolium et alibi. Item quidquid acquisivimus a domino de Ceuseyo ${ }^{\mathrm{m}}$ apud Montem Layin et alibi. Item quasdam terras dictas vulgariter Les Ers$\mathrm{sat}^{\mathrm{n}}$ de Coldreyo sitas in finagio Laiginario cum sexaginta sex bichetis frumenti annui redditus assignatis et assessis super gueargnagium ${ }^{\circ}$ domus dicte Lavataille et pertinentiarum dicte domus, quas terras et quem frumenti redditum acquisivimus a Iohanne de Anceyo franco armigero. Item quidquid acquisivimus a domino de Tanlayo et eius uxore et a Iohanne dicto de Bone Enure armigero apud villas dictas Vertau et Lemeisniz et in finagiis et pertinentiis earundem. Item stagnum magnum de Laigniaco et nemus nostrum de Essargelent. Item molendina cum feodis eorundem, ripparia et pratis nostris de Laigniaco. Item omnimodam iustitiam et dominium predictorum locorum omnium et pertinentiarum eorundem, salvis nobis et successoribus nostris comitibus Tornodori conditione predicta de supradictis malefactoribus tradendis et liberandis preposito nostro de Tornodoro, qui pro tempore fuerit, vel locum eius tenenti, sicut superius est ${ }^{\mathrm{p}}$ expressum, ac etiam alta iustitia in aliis hereditagiis ${ }^{q}$ hospitali predicto concessis a nobis sitis tam apud Laigniacum et apud

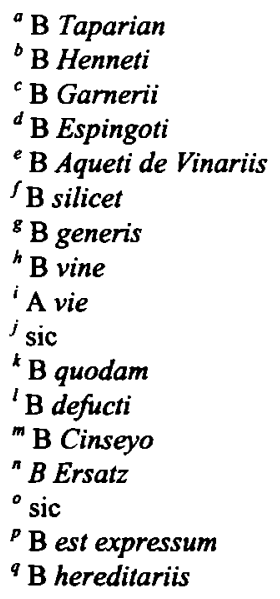


Tornodorum extra metas hospitalis predicti quam in finagiis de Tornodoro et Laigniaco predictis necnon in venatione in nemoribus supradictis. Volumus etiam et ordinamus ${ }^{\mathbf{a}}$ homines dicti hospitalis temporibus tam guerre quam pacis ab omni $i^{b}$ exercitu et chevaucheria ${ }^{c}$ liberos esse penitus ${ }^{d}$ et immunes, nisi tamtummodo pro defensione speciali comitatus Tornodori, si, quod absit, inimici ad exercitum contra eundem venirent, nam tunc ipsum comitatum defendere tenerentur sicut alii homines nostri de comitatu predicto. Item volumus, quod magister dicti hospitalis iurisdictionem et iustitiam familie sue et pertinentiarum eiusdem hospitalis ac morantium in eisdem habeat, alta tamen iustitia nobis salva et successoribus nostris dominis Tornodori, nisi tantum ${ }^{e}$ in locis, in quibus dictum hospitale altam habet iustitiam et habebit. Damus iterum eidem hospitali ius habendi et tenendi perpetuo in foresta et usagiis de Mauna singulis annis quadraginta porcos libere et sine pasnagio ${ }^{\mathrm{f}}$ et alia quacumque redebintia persolvenda. Iterum usuarium in dictis usagiis ad ${ }^{\mathrm{g}}$ edificandum et ardendum et ad omnia alia necessaria hospitalis predicti et pertinentiarum eiusdem, si sufficerint ${ }^{\mathrm{h}}$, alioquin, si non sufficerint ${ }^{\mathrm{i}}$, in foresta de Mauna, super qua insufficientia ${ }^{j}$ volumus dicto magistro per simplex verbum suum credi et fidem eidem plenius adhiberi. Item volumus in presenti fundatione dicti hospitalis et ordinamus, quod, si nos eodem hospitali non sufficienter completo mori contingerit ${ }^{k}$, executores nostri vel duo aut unus ipsorum cum magistro seu instituti ad hoc ${ }^{1}$ ab eisdem executoribus vel duobus ipsorum dictum hospitale cum pertinentiis suis edificare et perficere valeant et debeant de bonis nostris et hospitalis predicti ita videlicet, quod ipsi executores vel duo aut unus ipsorum cum magistro seu dicti instituti de exitibus dictorum bonorum eiusdem hospitalis levare ${ }^{\mathrm{m}}$ possint et levent quolibet anno usque ad quinque annos a die obitus nostri numerandos quingentas libras Turonensium parvorum convertendas in dicti hospitalis edificationem et perfectionem absque contradictione et impedimento dictorum magistri et fratrum et alterius cuiuscumque. Et restringantur propter hoc expense et missiones dicti hospitalis per executores nostros vel duos ipsorum, prout expediet et decebit. Preterea, cum nos ultra predicta et

\author{
${ }^{a} \mathrm{~B}$ hordinamus \\ ${ }^{b} a b$ omni fehlt B \\ ${ }^{c} \mathrm{~B}$ chuaucheya \\ ${ }^{d} \mathrm{~B}$ peratus \\ 'B tamen \\ ${ }^{f} \mathrm{~B}$ passnagio, $\boldsymbol{n}$ von passnagio interlinear \\ ${ }^{8} \mathbf{B}$ et \\ ${ }^{h}$ A suffecerint \\ 'A suffecerint \\ ${ }^{j} \mathrm{~B}$ insuficientia \\ ${ }^{k}$ A contigerit \\ 'B hec \\ ${ }^{m}$ A levari
}


alia, que contulimus dicto hospitali, proponamus ${ }^{a}$ augmentare $^{b}$ facultates hospitalis prefati de trecentis libratis Turonensium annui redditus, volumus et ordinamus, quod, si dicti redditus casu aliquo impedimentum prestante non acquirentur eidem hospitali in vita nostra, executores nostri seu aliquis eorum cum magistro dicti hospitalis aut magister ipsis cessantibus pro eodem hospitali in comitatu ${ }^{\mathfrak{c}}$ Tornodori videlicet in feodis, retrofeodis seu censivis dicti comitatus libere sine laudis et vendis possit acquirere trecentas ${ }^{d}$ libratas Turonensium annui redditus et quod heredes et successores nostri in dicto comitatu seu causam ab ipsis habentes teneantur et sint obligati ad patiendum et substinendum, quod dicta acquisitio fiat et perficiatur usque ad summan predictam absque eo, quod dictum hospitale compellant ${ }^{e}$ redditus ipsos, bona seu res acquisitas ponere extra manum suam, et redditus predictos sive res et bona, quecumque sint, de quibus prefati redditus percipiendi erunt, dicto hospitali penitus admortizare teneantur. Et nos etiam, quantum possumus, ex nunc admortizamus salvo iure dicto hospitali acquirendi in dicto comitatu res et bona, que acquirere poterit ultra predicta de iure communi. Item volumus, ut persone dicti hospitalis per totum comitatum Tomodori eant, redeant, ducant et reducant res suas, vendant et emant ad usus proprios per se et suas familias sive in mercatis et nundinis sive extra libere absque onmi redebentia et ${ }^{f}$ exactione vinagii, minagii, passagii, pedagii, rotagii et thelonei aliaque exactione quacumque, nec ab hiis ${ }^{\mathrm{g}}$ vel ab eorum nuntiis occasione exactionum huiusmodi aliquid exigatur, sed a talibus in posterum sint immunes, quantum ad ius nostrum speciale et successorum nostrorum pertinet et etiam pertinebit. Nos autem res et bona omnia supradicta a nobis eidem hospitali data et concessa plenius admortizamus pro hospitali predicto et ea volumus et ordinamus pro eodem esse et manere in perpetuum admortizata sine cohertione aliqua ${ }^{\mathrm{h}}$ extra manum suam ponendi aliqua de causa vel occasione quacumque et ea omnia ad ipsum hospitale ex hoc nunc in perpetuum iure hereditario pertinere, salvis et retentis nobis et successoribus nostris comitibus Tornodori garda hospitalis predicti, ut premissum est, et resorto in temporalitate eiusdem et pertinentiarum ipsius. Item volumus, quod magister et fratres hospitalis predicti una cum executoribus vel aliquo seu aliquibus executoribus nostris, si vixerint, quecumque de predictis omnibus permutare vel escambire voluerint, permittent vel escambient libere, prout utilitati dicti hospitalis viderint expedire, super quibus es-

\footnotetext{
A folgt uita comite, B uita coite (?)

${ }^{b} \mathrm{~B}$ aumentare

${ }^{c}$ B coitalu

${ }^{d} \mathrm{~B}$ trescentas

'B compellatur

$f_{\text {et }}$ fehlt B

${ }^{8} \mathrm{~B}$ iis

${ }^{h} \mathrm{~B}$ aliquam
} 
cambio seu permutatione bonorum, edificatione et perfectione dicti hospitalis et pertinentiarum eiusdem magistro et fratribus ac executoribus et eorum cuilibet concedimus ${ }^{a}$ plenariam et liberam potestatem. Et ea, que ex causa escambii seu permutationis huiusmodi fuerint acquisita, hospitali predicto eidem ex hoc nunc admortizamus et admortizata esse volumus sicut et alia, que eidem dedimus, prout superius est expressum. Et licet visitationis causa, quandoque prelato procuratio debeatur, nos tamen et, si dictum hospitale seu domum Dei a domino .. Lingonensi episcopo, qui pro tempore erit, vel eiusdem vices gerentibus visitari, quandoque contingerit ${ }^{b}$, volumus supplicando cum onerato, superaddi pondus non debeat ipsum hospitale pauperum a refectione seu procuratione huiusmodi quietum manere penitus et immune. Item volumus, quod prefatus magister, quoad vixerimus, coram nobis vel mandato nostro et post mortem nostram coram mandato dicti comitis Tornodori vocatis duobus capellanis et magistra hospitalis predicti ac duobus burgensibus Tornodori probis viris per dictum comitem Tornodori eligendis bis quolibet anno videlicet in ieiuniis veris et autumpni ${ }^{c}$ per se vel per alium reddere debeat compotum et rationem administrationis sue. Item volumus et ordinamus pro statu et libertate hospitalis predicti, ut ipsius bona illesa et in usus pauperum secundum nostrum desiderium convertantur et applicentur, quod nullus heredum et successorum nostrorum seu causam ab ipsis habentium aliquam molestiam, inquietationem, perturbationem, iacturam, gravamen seu onus ${ }^{d}$ per se vel quemcumque alium inferat vel inferre valeat hospitali predicto et suis pertinentiis quibuscumque servientes vel quoscumque alios, canes vel equos ibi mittendo ad quiescendum et morandum et pastum habendum vel aliter quocumque modo in diminutionem bonorum dicti hospitalis vel quecumque alia gravamina, quocumque nomini censeantur, inferre valeat, ne fraudis alicuius adinventione bona dicti hospitalis diminui valeant seu etiam defraudari. Ordinamus etiam et volumus, quod Guillelmus comes Autissiodorensis ${ }^{e}$ successor noster in comitatu Tornodori carissimus nepos noster ac etiam successores eiusdem et ab ipso causam habentes et etiam habituri in comitatu Tornodori predicto et quicumque domini Tornodori, qui ${ }^{\mathrm{f}}$ pro tempore fuerint, quamprimum ad ipsos dominium seu comitatus Tornodori obveniet, et ballivus et prepositus Tornodori in institutione sua iurent et iurare debeant ac etiam teneantur super sancta Dei evangelia magistro dicti hospitalis vel eius mandato seu procuratori ad requisitionem ipsius magistri vel mandati seu procuratoris eiusdem, antequam

\footnotetext{
a concedimus fehlt A und B

${ }^{b}$ A contigerit

${ }^{c}$ sic

${ }^{d} \mathrm{~B}$ hous

e B Autisiodorensis

${ }^{f}$ qui fehlt A und B
} 
ipsi domini levent aliquid vel levari faciant de redditibus et exitibus dicte ville Tornodori, quod ipsi fundationem, dona, gardas, custodias et libertates omnes et singulas dicti hospitalis et pertinentiarum eiusdem, prout verbo ad verbum presentibus litteris plenius continetur, in quantum ad eos pertinet et etiam pertinebit, in omnibus et per omnia intergraliter adimplebunt et inviolabiliter observabunt et quod contra per se vel per alium seu alios non venient in futurum nec sustinebunt ${ }^{\mathrm{a}}$ pro posse suo, quod ab aliquo vel ab aliquibus contra premissa fiat aliquid seu etiam attemptetur ${ }^{b}$. Quod, si domini predicti vel aliquis eorum iuramentum predictum elapso mense a tempore requisitionis predicte facere seu prestare, quod absit, distulerint vel recusaverint, ordinamus et volumus, quod ex tunc redditus et exitus omnes dicte ville Tornodori fiant et sint hospitalis predicti, quos ipsum hospitale percipiat et habeat et ex eis faciat integre fructus suos, quousque predictum super premissis a domino prestitum seu factum, ut dictum est, fuerit iuramentum. Qui dominus dictos fructus sic levatos repetere vel repeti facere per dictum iuramentum non poterit nec debebit nec eos magister et fratres eiusdem hospitalis restituere dicto domino tenebuntur. Ad que omnia prestanda et adimplenda ipsos per excellentissimum dominum regem Francie illustrem ad requisitionem magistri $\operatorname{dicti}^{c}$ hospitalis compelli requirimus supplicantes intuitu pietatis. Magister vero dicti hospitalis ad minus per sex menses per annum in dicto hospitali residentiam facere tenebitur personalem, nisi forsitan iusta ${ }^{d}$ causa et honesta fuerit impeditus. Volumus autem et etiam ordinamus, quod Robertus de Lusarche fidelis clericus et familiaris noster, qui dicti hospitalis promotionem desiderat et de cuius fidei integritate confidimus, dictum hospitale primo fundationis loco regat, dispenset ac etiam administret, et ipsum primum magistrum, dispensatorem ac rectorem instituimus et etiam ordinamus. Ordinationi autem habitus et indumentorum, de quibus superius ordinavimus, ipsum nolumus subiacere nec astringi ${ }^{e}$. Quam ordinationem ab eius successoribus magistris dicti hospitalis inconcusse volumus observari, salvo tamen quod magistri, qui fratres non fuerint, pennas alias, quam superius ordinavimus, quascumque voluerint, in suis vestibus habeant de suo proprio persolvendas. Volumus etiam, quod ipse Robertus magister et successores sui magistri dicti hospitalis beneficia ecclesiastica et bona sua quecumque alia proprie valeant obtinere, nisi fuerint de capellanis eiusdem hospitalis assumpti. Et, ut ista ${ }^{\mathrm{f}}$ fundatio et ordinatio dicti hospitalis robur perpetue firmitatis obtineat, nos ad hoc dictum Guillelmum heredes et successo-

\footnotetext{
${ }^{a} \mathrm{~B}$ substinebunt

${ }^{b} \mathrm{~B}$ attentetur

${ }^{c} \mathrm{~B}$ dicti magistri

${ }^{d} \mathrm{~B}$ iuxta

${ }^{e} \mathrm{~A}$ adstringi

${ }^{f} \mathrm{~B}$ iuxta
} 
res nostros Tornodori dominos successive una cum terra et bonis comitatus Tornodori plenius obligantes presentes litteras fecimus sigilli nostri munimine roborari. Actum anno Domini millesimo ducentesimo nonagesimo tertio ${ }^{a}$, dies iovis post octavas ${ }^{\mathrm{b}}$ Pasche $^{2}$.

Nulli ergo omnino hominum liceat hanc paginam nostre suppletionis et confirmationis infringere vel ei ausu temerario contraire. Si quis autem hoc attemptare presumpserit, indignationem omnipotentis Dei et beatorum Petri et Pauli apostolorum eius se noverit incursurum ${ }^{c}$. Datum apud Urbemveterem, nonis septembris pontificatus nostri ${ }^{\mathrm{d}}$ anno tertio.

${ }^{1}$ Karl I. von Anjou, König von Sizilien, † 7.1.1285.

${ }^{2} 1293$ April 9.

\footnotetext{
${ }^{a} \mathrm{~B}$ CCLXXXXIII

${ }^{b} \mathrm{~B}$ octabas

${ }^{c} \mathrm{~B}$ Nulli ergo etc. nostre supplectionis confirmationis etc. ${ }^{d}$ pontificatus nostri fehlt $\mathrm{B}$
} 